\title{
ELEMENTOS BÁSICOS PARA LA PROTECCIÓN DE LAS AGUAS SUBTERRÁNEAS APLICADOS EN EL ACUÍFERO DE MANAGUA, NICARAGUA
}

\author{
Arcadio Choza \\ Proyecto SUWaR/MARENA (1993-2001), Managua, Nicaragua \\ Miembro de GWP-Centroamérica (Nicaragua, 2000-2002). \\ E-Mail: Choza@alfanumeric.com.ni
}

\begin{abstract}
With the effort and coordination between several public institutions of Nicaragua and Sweden, it has been possible to contribute substantially to the protection of groundwater with the development of methods in 3 large aspects.

A technical frame developed in MARENA permitted the application of methods to locate the vulnerable areas to contamination in the aquifer and estimate the potential danger of anthropogenic activities to the groundwater. A communication frame developed at UNI, was used for the identification of obstacles for the protection of groundwater and identification of the interested parties. This frame can be complemented with education and publicity of the information about the subject. A complementary support frame, consisting of a geographic information system (GIS) and developed in MARENA is applied to make technical maps and to combine different elements, depending on the evaluation purposes. Also, a modeling system installed in ENACAL for the application of a mathematical FLOWPATH model was used to model the recharging areas of the fields with wells in the eastern sub-basin.

All these elements have been combined to produce a protection plan which contains the instructions about countermeasures that might be applied for the prevention and control of contamination of underground water in the aquifer of Managua and how to extend investigations to other aquifers in Nicaragua.
\end{abstract}

RESUMEN: Con el esfuerzo conjunto y coordinado de varias instituciones nicaragüenses y suecas se ha logrado contribuir sustancialmente a la protección de las aguas subterráneas por medio de la implementación de tres aspectos:

Un marco técnico desarrollado en MARENA que ha permitido la aplicación de métodos para conocer las áreas vulnerables a la contaminación en el acuífero y para estimar el peligro potencial para las aguas subterráneas que implican las actividades antropogénicas. Un marco de comunicación desarrollado en la UNI, que involucra la identificación de barreras a la protección del agua subterránea y de las partes interesadas. Un marco de apoyo complementario, compuesto de un sistema de información geográfica (SIG), desarrollado en MARENA para la elaboración de los mapas técnicos y la combinación de los diferentes elementos según el propósito de la evaluación.

Todos estos elementos se han combinado entre sí para producir un plan de protección que contiene las directrices sobre medidas que se pueden aplicar para la prevención y el control de la contaminación de las aguas subterráneas del acuífero de Managua y la extensión del trabajo a otros acuíferos del país. 


\section{ESTRATEGIA DE PROTECCIÓN DE LAS AGUAS SUBTERRÁNEAS}

Considerando la contaminación masiva a que están siendo sometidas las aguas superficiales en muchos países de la región, las aguas subterráneas se convierten en una fuente inestimable de agua de calidad aceptable para enfrentar los déficit de disponibilidad. Las principales barreras para lograr una efectiva protección de las aguas subterráneas se han identificado como falta de conciencia y conocimiento del problema, unida a una carencia de voluntad política e instrumentos regulatorios de protección.

En un esfuerzo conjunto y coordinado por el Ministerio del Ambiente y Recursos Naturales (MARENA), la Empresa Nicaragüense de Acueductos y Alcantarillados (ENACAL) y la Universidad Nacional de Ingeniería, con el apoyo técnico del Real Instituto de Tecnología (KTH) y la Universidad de Linköpin (Suecia) y el apoyo financiero de la Agencia Sueca para el Desarrollo Internacional (ASDI) se ha logrado contribuir sustancialmente a la protección de las aguas subterráneas con la implementación de tres aspectos.

\section{Área de estudio}

El acuífero de Managua es la principal fuente de abastecimiento para 1,3 millones de habitantes de la ciudad de Managua, que hasta 1999 tenía una población de 1907060 (INEC, 2001). El rápido crecimiento poblacional en combinación con las diferentes fuentes de contaminación, tanto puntuales como difusas, ha agravado la situación del agua durante los últimos 15 años. El área de trabajo es de aproximadamente $1040 \mathrm{~km}^{2}$ y está caracterizada por una estratificación compleja de materiales entre ellos, piroclastos, cenizas, flujos de lavas y depósitos aluviales.

El potencial anual de agua subterránea del acuífero de Managua comprendido en la cuenca sur del lago de Managua se estima en el orden de 158 MMC de los cuales 22,59 MMC se almacenan en la subcuenca occidental, 31,65 MMC en la subcuenca central y 104,27 MMC en la sub-

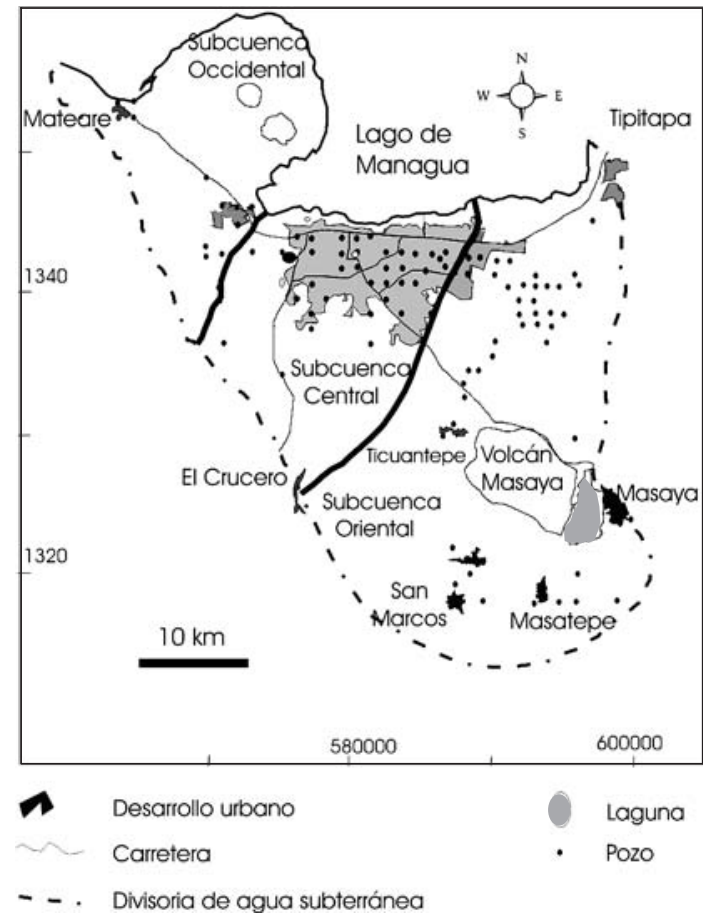

Fig. 1: Mapa del acuífero de Managua y las tres subcuencas en que se divide con sus principales campos de pozos de abastecimiento.

cuenca oriental (Fig. 1). Sin embargo, la subcuenca Central ya presenta condiciones de sobreexplotación y la subcuenca oriental reúne cinco campos de pozos con 50 MGD, y para el año 2001 entró en funcionamiento el Campo de Pozos Managua II con una extracción de 16 MGD (INAA-JICA, 1993).

\section{Metodologías desarrolladas}

El desarrollo de métodos para la estrategia de protección del agua subterránea consiste básicamente en los siguientes componentes básicos:

Vulnerabilidad hidrogeológica.

- Caracterización del peligro potencial de contaminación de las fuentes puntuales y difusas.

- Riesgo potencial de contaminación del agua subterránea. 
Además, se desarrollaron como elementos auxiliares, la estimación del valor relativo de la protección del agua subterránea, la protección de pozos de agua potable y la identificación de barreras para la implementación del plan de protección. En el presente artículo solo se abordan los primeros tres elementos. En la figura 2 se presenta de manera esquemática la conceptualización de la estrategia de protección del acuífero de Managua.

\section{Vulnerabilidad hidrogeológica}

La vulnerabilidad hidrogeológica se define como la sensibilidad natural que tiene un acuífero a la contaminación, lo que la convierte en una herramienta importante para la formulación de un plan de protección de aguas subterráneas. Por medio del método DRASTIC es posible cuantificar la vulnerabilidad de un acuífero. Este método fue desarrollado por la Agencia de Protección Ambiental de los Estados Unidos (EPA) y ha sido empleado en diez condados de los Estados Unidos para elaborar los mapas de vulnerabilidad hidrogeológica (Aller et al., 1987).

Antes de adoptar el DRASTIC, el proyecto realizó una revisión de los diferentes métodos que para éste propósito han sido utilizados y se estimó que este método, por su moderada complejidad, resulta apropiado para aplicarlo a las condiciones de Nicaragua y puede ser fácilmente reproducible en cualquier otro acuífero del país. La metodología DRASTIC es un sistema estandarizado para evaluar la sensibilidad natural de un acuífero a la contaminación ante la presencia de una carga contaminante depositada, utilizando escenarios hidrogeológicos con características comunes y por consiguiente con una vulnerabilidad común a la contaminación.

El método se apoya en el análisis de siete parámetros a los cuales se les aplica un sistema numérico que permite ponderar la intervención que tiene el parámetro en el análisis de la vulnerabilidad. Los siete parámetros fueron ordenados para formar el nombre del método con sus siglas en inglés (Fig. 3).

$\begin{array}{lll}\text { D } & \text { Depth to Water } & \text { Profundidad del Agua } \\ \text { R } & \text { Net Recharge } & \text { Recarga Neta } \\ \text { A } & \text { Acuifer Media } & \text { Medio del Acuífero } \\ \text { S } & \text { Soil Medio } & \text { Medio del Suelo } \\ \text { T } & \text { Topography } & \text { Topografía } \\ \text { I } & \text { Impact on the vadose } & \text { Impacto de la zona } \\ & \text { zona } & \text { vadosa } \\ \text { C } & \text { Hydraulic Conductivity } & \text { Conductividad hidráulica }\end{array}$

La aplicación de la metodología se realizó a través de un sistema numérico que consta de tres partes principales: pesos, escalas y clasificación. Haciendo uso de estos tres elementos

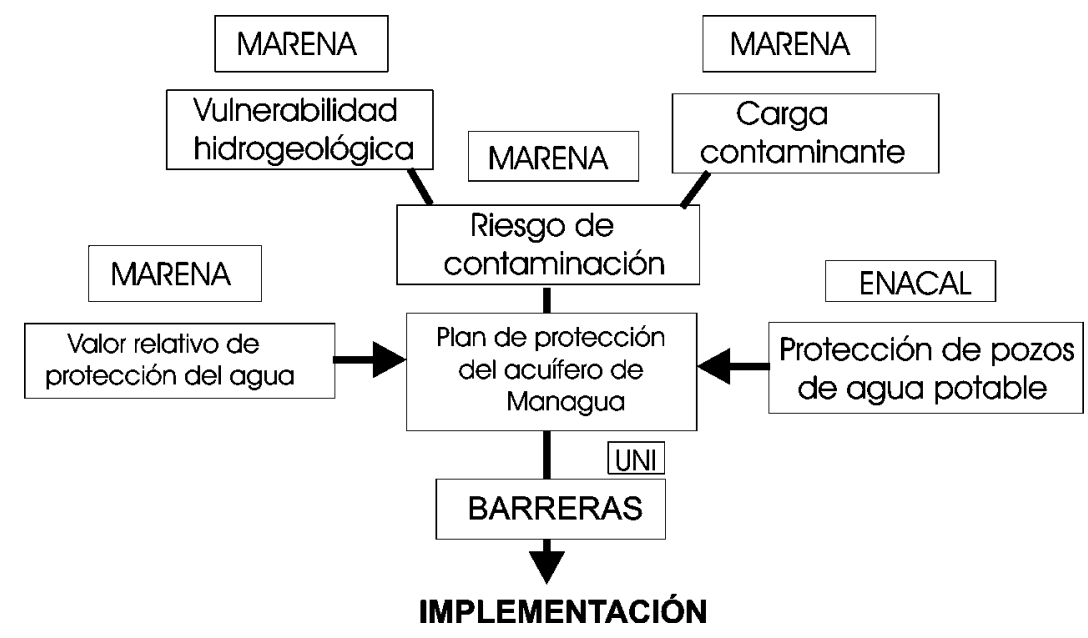

Fig. 2: Esquema conceptual de la estrategia de protección del acuífero de Managua. MARENA: Ministerio del Ambiente y Recursos Naturales. ENACAL. Empresa Nicaragüense de Acueductos y Alcantarillados. UNI: Universidad Nacional de Ingeniería. 


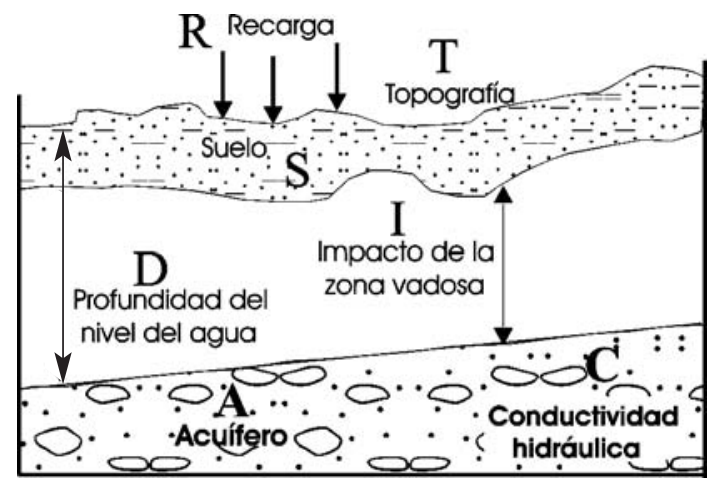

Fig. 3: Esquema de interpretación de los parámetros utilizados en el método DRASTIC.

evaluativos, se puede llegar a obtener el valor numérico DRASTIC (VND) y posteriormente el índice DRASTIC (SUWaR/NICARAGUA, 2000a).

Peso: es el valor numérico relativo asignado a cada parámetro en relación a su importancia con respecto a otro, en el proceso de determinación de la vulnerabilidad (Cuadro 1).

Escalas: rangos en que se divide a cada parámetro según sus condiciones físico-naturales y el impacto de estas en la sensibilidad natural del acuífero.

Clasificación: valor numérico asignado a cada uno de los rangos en que se ha dividido al parámetro.

A manera de ejemplo, se presentan en el cuadro 2 las escalas y clasificación para el parámetro de profundidad del agua analizado y para la recarga neta (Cuadro 3), el resto de parámetros pueden ser consultados en Aller et al., (1987).

Cuadro 1

Parámetros DRASTIC y pesos asignados

\begin{tabular}{lc}
\hline Parámetro & Peso asignado \\
\hline Profundidad del agua & 5 \\
Recarga neta & 4 \\
Medio del acuífero & 3 \\
Suelo & 2 \\
Topografía & 1 \\
Impacto de la zona vadosa & 5 \\
Conductividad hidráulica & 3 \\
\hline
\end{tabular}

Cuadro 2

Escala y clasificación para la profundidad del agua

\begin{tabular}{cc}
\hline Escala $(\mathrm{m})$ & Clasificación \\
\hline $0-1.5$ & 10 \\
$1.5-9$ & 9 \\
$9-15$ & 7 \\
$15-23$ & 5 \\
$23-30$ & 3 \\
$>30$ & 1 \\
Peso: 5 & \\
\hline
\end{tabular}

Cuadro 3

Escala y clasificación para la recarga neta (mm/año)

\begin{tabular}{cc}
\hline Escala & Clasificación \\
\hline $0.0-50$ & 1 \\
$50-100$ & 3 \\
$100-180$ & 6 \\
$180-250$ & 8 \\
$>250$ & 9 \\
Peso: 4 & \\
\hline
\end{tabular}

Valor Numérico DRASTIC (VND): el valor de calificación asignado para cada rango de la escala, multiplicado por el peso, permite obtener el valor numérico DRASTIC del parámetro.

Indice DRASTIC (ID): finalmente, una vez abordados los 7 mapas (uno por cada parámetro), a través del proceso de sobreposición, se van identificando las áreas que constituirán un escenario definido por siete parámetros. La suma de los siete valores numéricos DRASTIC, proporciona el índice DRASTIC del escenario. Este índice es sólo un instrumento de evaluación relativa y no está supuesto a dar respuestas absolutas. En términos matemáticos, el índice DRASTIC se expresa así:

$$
\begin{gathered}
\mathrm{DrDw}+\mathrm{RrRw}+\mathrm{ArAw}+\mathrm{SrSw}+\mathrm{TrTw}+\mathrm{IrIw}+\mathrm{CrCw} \\
\mathrm{r}=\text { Clasificación } \\
\mathrm{w}=\text { Peso }
\end{gathered}
$$


Los índices DRASTIC se obtuvieron de las sumas de los valores numéricos para cada escenario. Luego de realizar las sobreposiciones de las variables DRASTIC y, de acuerdo a los criterios antes expresados, se obtuvo el mapa de escenarios hidrogeológicos, proporcionando al usuario una cuantificación de los principales factores geológicos e hidrogeológicos que contribuyen en la determinación del grado de vulnerabilidad relativa que tiene un área ante una posible carga contaminante (SUWaR/MARENA, 2000a).

Para el caso del Acuífero de Managua se realizó una agrupación de los escenarios, la cual no está incluida en el método original, para obtener un mapa simplificado que funcione como instrumento de planificación (Fig. 4). Este se caracteriza por ser sencillo, de fácil uso, utiliza la información existente y permite que personas con diferentes formaciones y niveles académicos puedan hacer uso de él. Esta agrupación permite obtener escenarios hidrogeológicos de mayor comprensión espacial con base en los siguientes criterios:

a-

Se agruparon escenarios con características geológicas e hidrogeológicas muy similares y con baja diferencia en sus índi-

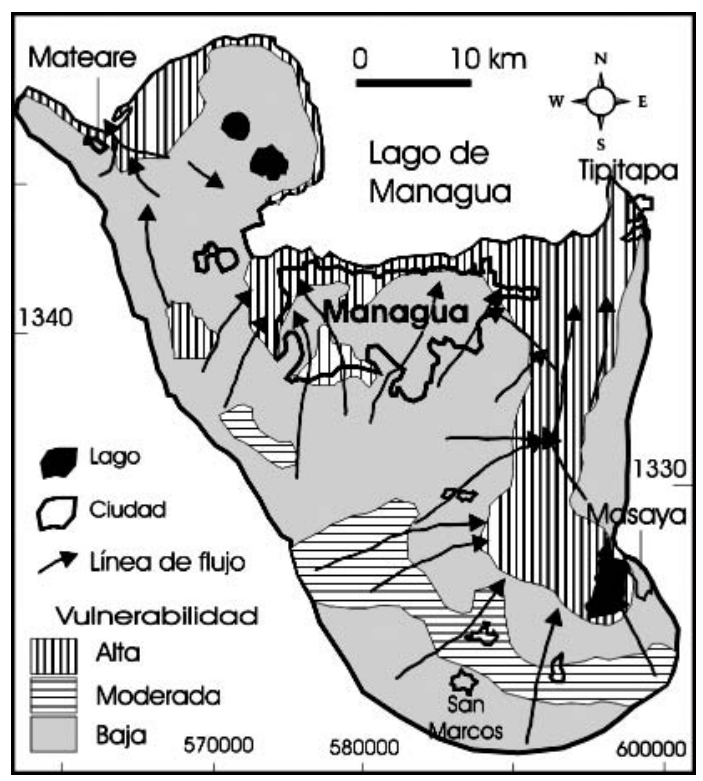

Fig. 4: Mapa de vulnerabilidad a la contaminación del agua subterránea desarrollado en el proyecto usos sostenible de los recursos hídricos. ces DRASTIC desde el punto de vista cualitativo de las condiciones geológicas e hidrogeológicas.

b- $\quad$ Se agruparon escenarios con igual índice DRASTIC.

Cada zona de vulnerabilidad quedó compuesta por uno ó más escenarios hidrogeológicos con diferencias que están definidas por las condiciones tanto geológicas como hidrogeológicas propias de un acuífero de origen volcánico y de carácter freático. Finalmente, con el propósito de lograr una aplicación cualitativa del índice DRASTIC, se elaboró una escala de rangos de vulnerabilidad considerados como alto, moderado y bajo. El cuadro 4 presenta los rangos de vulnerabilidad propuestos por el proyecto.

Cuadro 4

Rangos de vulnerabilidad

\begin{tabular}{ll}
\hline APRECIACIÓN CUALITATIVA & Índice \\
\hline a) Área de alta vulnerabilidad & 140 a $>200$ \\
b) Área de moderada vulnerabilidad & $\begin{array}{l}100-139 \\
<99\end{array}$ \\
c) Área de baja vulnerabilidad & $<9$ \\
\hline
\end{tabular}

Fuente: SUWaR/MARENA, (2000a)

\section{RESULTADOS}

\section{Áreas de vulnerabilidad hidrogeológica}

\section{Área de "alta vulnerabilidad"}

El área de alta vulnerabilidad quedó constituida por ocho escenarios hidrogeológicos y se caracteriza por aquellas zonas donde los perfiles naturales del suelo están compuestos por materiales porosos, permeables, reducido espesor del suelo con alta permeabilidad, escasa profundidad del agua y alto grado de fracturamiento. Estas características facilitan el paso a cualquier tipo de contaminante depositado sobre y debajo de la superficie del suelo en un tiempo corto con respecto a las otras áreas de vulnerabilidad.

La pendiente topográfica de estos escenarios es de baja a moderada permitiendo la infiltración del agua y se le adiciona la presencia de 
cuerpos de aguas superficial (laguna de Masaya, Asososca, Nejapa) que son de mucha importancia en el área de estudio. La laguna de Masaya contribuye a la recarga natural del acuífero localizado en la subcuenca Oriental a través de la conexión hidráulica que tiene con el manto acuífero. La laguna de Asososca aporta parte de su caudal para el abastecimiento de agua potable de un sector de la población de Managua, por tanto es de vital importancia su protección.

La presencia de las fallas Cofradía, Veracruz, Aeropuerto, Estadio, Tiscapa y todas aquellas fallas y fracturas (las denominadas fallas bailarinas) de menor escala ubicadas en la subcuenca central y occidental, jugaron un papel importante en la calificación del alto grado de vulnerabilidad en éste ambiente hidrogeológico (SUWaR/MARENA,2000a).

\section{Área de "Moderada Vulnerabilidad"}

El área de moderada vulnerabilidad, está constituida por tres escenarios y se caracteriza por zonas donde los materiales geológicos presentes son piroclastos sueltos, ocurre un fracturamiento moderado, espesores de suelo mayores de $90 \mathrm{~cm}$ con moderado contenido de materia orgánica. Además, la permeabilidad es de moderada a moderadamente alta, permitiendo el paso a contaminantes móviles y persistentes en un mediano plazo con relación al área de alta vulnerabilidad.

La zona de moderada vulnerabilidad es la de mayor extensión en el área de estudio (Fig. 4). Los escenarios de esta área están ubicados en la deposición geológica de la Formación Las Sierras localizada en la subcuenca central y oriental, en un pequeño sector que limita la caldera de Masaya, y también en los materiales cuaternarios aluviales ubicados en la península de Chiltepe, los Brasiles y ciudad Sandino (SUWaR/MARENA,2000a).

\section{Área de "Baja Vulnerabilidad"}

Esta área quedó caracterizada por valores de índices DRASTIC, de 89 y 98. Entre ambos escenarios hidrogeológicos existe una diferencia de las condiciones geológicas que se da por la presencia de un lente limo-arcilloso y por una colada de lava andesítico-basáltica que funcionan como barreras de atenuación o retardación de contaminantes. Estos materiales litológicos se encuentran en la zona no saturada, lo que significa que el parámetro de mayor influencia es el impacto de la zona vadosa.

La gran profundidad de las aguas subterráneas, la presencia de material litológico como elemento atenuante de la carga contaminante, baja conductividad hidráulica, altas pendientes topográficas y gruesos espesores de suelo, contribuyen positivamente a la atenuación de la posible carga contaminante (SUWaR/NICARAGUA 2000a). Se estima que esta área de baja vulnerabilidad es solo sensible a contaminantes móviles y persistentes y en tiempos relativamente largos con respecto a las otras áreas.

\section{Usos del mapa de vulnerabilidad}

El mapa de vulnerabilidad hidrogeológica, es un instrumento auxiliar para orientar adecuadamente las decisiones relacionadas con la protección de las aguas subterráneas del acuífero analizado. No obstante la vulnerabilidad no es el único criterio para una toma de decisión con respecto a la protección. Este conocimiento, combinado con el peligro potencial de las fuentes de contaminación y con el valor relativo del agua, permiten establecer una estrategia de protección más adecuada. Sin embargo, es muy importante señalar, que en ausencia de una evaluación de la carga contaminante y del valor relativo del agua, la estimación de la vulnerabilidad hidrogeológica sigue siendo un instrumento muy útil para:

- Priorizar áreas donde la protección del agua subterránea es de vital importancia.

- Identificar áreas donde se justifique una atención especial o esfuerzos encaminados a la protección del agua subterránea.

- Utilizarlo como parte de una estrategia en la identificación de áreas donde se recomienden medidas de protección adicionales o menos rigurosas para la colocación de tanques de almacenamiento subterráneos.

- Identificar áreas donde los plaguicidas representen una mayor amenaza para el agua subterránea. 
- Priorizar áreas con fines de monitoreo.

- Identificar características hidrogeológicas especiales que por lo general influyen sobre las actividades de descontaminación.

- Identificación de vacíos en los datos que afecten la evaluación del potencial de contaminación.

- Para la justificación de estudios hidrogeológicos más detallados.

- Como instrumento de estudio para determinar si una instalación está ubicada (o se está ubicando) en un área vulnerable a la introducción de contaminantes en la superficie.

\section{Peligro potencial de contaminación}

En el acuífero de Managua existe una gran diversidad de actividades que pueden ser identificadas como posibles fuentes contaminantes tales como industriales, agrícolas, municipales y otras (gasolineras, aeródromos, hospitales), derivadas de la actividad antropogénica la cual es considerada como un factor de incidencia para el peligro potencial de contaminación de las aguas subterráneas. Por lo tanto, es muy importante conocer las características generales de los diferentes focos identificados en el área de estudio.

\section{Actividad industrial}

Hasta el año 1982 en Nicaragua existían 4553 industrias en todo el país, el $41 \%$ de ellas estaban situadas en la ciudad de Managua de las cuales el 1,32\% representan las 60 industrias que se encuentran en el área de la subcuenca oriental, considerando únicamente agroindustrias, producción agropecuaria (café, trillo de arroz), mediana y gran industria (SUWaR/ NICARAGUA, 2000b).

En esta zona se encuentran las industrias de mayor capacidad económica o la gran industria, existen industrias químicas, farmacéuticas, industrialización de carnes, bebidas (alcohólicas y no alcohólicas), fabrica de cigarrillos, cerillos, tostadurías, elaboración de galletas, almacenamientos industriales de hidrocarburos, algunas históricas como la PENWALT que fue cerrada por contaminación de mercurio y por emanacio- nes de gases (hipoclorito de sodio), fábrica de baterías y fábrica de agroquímicos.

\section{Actividad agrícola}

Las subcuencas oriental, occidental y parte alta de la central, se caracterizan por ser zonas eminentemente agrícolas, por la vocación de los suelos (agrícolas) y el alto porcentaje de estas actividades que se desarrollan en estas áreas. La mayor parte de estas actividades se concentran en los alrededores de los poblados de Sabana Grande, Veracruz, Cofradía, Zona de los Pueblos, El Crucero, Los Brasiles, Mateare, y todo el perímetro de la península de Chiltepe.

Históricamente en la parte baja de las subcuencas oriental y occidental, entre los poblados de Sabana Grande, Cofradía, ciudad Sandino, Mateare y los Brasiles, se llevó a cabo una agricultura intensiva del cultivo del algodón durante las décadas de los setenta y parte de los ochenta inclusive. En la parte alta de la subcuenca oriental, zona del Crucero y Los Pueblos, se ha desarrollado con gran intensidad el cultivo del café. En todo el perímetro de la península de Chiltepe se ha desarrollado una ganadería intensiva de forma tecnificada con el auge del proyecto Chiltepe, en la década de los ochenta. Actualmente los cultivos más importantes en el área son: el café, granos básicos, piña, pastos y ganadería y otros de menor importancia tales como hortalizas y frutales (SUWaR/NICARAGUA, 2000b).

\section{Actividades municipales}

El análisis de las actividades municipales ha considerado a aquellas áreas de desarrollo urbano con o sin alcantarillado sanitario, la disposición de desechos sólidos domésticos, la pequeña industria y talleres de servicio que son potencialmente contaminantes para el agua subterránea del acuífero de Managua.

Las áreas de desarrollo urbano y rural representan el 3\% aproximadamente del área del acuífero, siendo el área más representativa la ciudad de Managua. Es importante señalar que existe un crecimiento acelerado de nuevas áreas pobladas en los alrededores de las urbanizaciones ya existentes. Aunque la mayoría de ellas tienen los servicios básicos (agua potable y luz eléctrica); 
muchas de ellas no cuentan con los servicios de saneamiento (alcantarillado sanitario y recolección de basura).

En algunos de éstos poblados no se presta servicio de recolección de basura, por lo que los pobladores tienen que hacer su propio manejo y disposición final de la basura y desechos líquidos. Muchas casas o familias utilizan pozos excavados abandonados para depositar sus desechos sólidos y líquidos; lo que constituye un serio peligro de contaminación para las aguas subterráneas debido al proceso de lixiviación, además de poner en riesgo la salud de los pobladores.

Managua representa el $64 \%$ de las áreas de desarrollo urbano y rural de la zona de estudio. Posee una alta densidad poblacional ya que el 30\% de la población del país reside en ella. Después del terremoto de 1972, ha sido característico de la ciudad una expansión desordenada. Si a esto se le agrega la falta de un Plan de Desarrollo Urbano en esos años, tenemos como resultado que muchas urbanizaciones se encuentran ubicadas en sitios inadecuados (fallas geológicas, bajo líneas del tendido eléctrico, en zonas de inundación, a la orilla de cauces, etc.) y en lugares en donde carecen de los servicios públicos básicos y de saneamiento (SUWaR/NICARAGUA,2000b).

Managua cuenta con un sistema de alcantarillado sanitario convencional. La red cubre cerca del $65 \%$ de la ciudad, pero sirve únicamente a un 50 a $55 \%$ de la población. El sistema presenta serios problemas por el bajo nivel de cobertura, los desagües en la ribera y la falta de tratamiento de las agua negras que son vertidas al lago de Managua (Abt, 1995).

Puede decirse que aunque existe una red de alcantarillado sanitario en las ciudades de Managua y Masaya, es muy probable que existan fugas de aguas residuales domésticas a lo largo de toda la red debido a fisuras ocasionadas por la antigüedad del sistema, el tipo de material utilizado por las tuberías y la sismicidad de la zona. Esto se suma a la descarga proveniente del resto de áreas de desarrollo urbano que no cuentan con este servicio.

En Managua, el manejo de los desechos sólidos se ha convertido en uno de los problemas ambientales más relevantes. La Alcaldía de Managua ha establecido puntos de recolección de basura en aquellas zonas que no están cubiertas por el servicio de recolección domiciliar. Sin embargo, existen innumerables basureros ilegales en toda la capital, cuyas características más notables son su temporalidad y un control nulo del tipo de desechos que ahí se depositan. Otro lugar de disposición de basura, aún en zonas que cuentan con el servicio de recolección a domicilio, son los cauces de drenaje pluvial. Muchos de ellos no poseen revestimiento a lo largo de algunos puntos de la capital.

\section{Desarrollo metodológico}

El procedimiento utilizado para conocer la carga contaminante derivada de las actividades anteriormente inventariadas, consiste en evaluar el peligro potencial de contaminación que representa cada foco contaminante para las aguas subterráneas. El análisis realizado para la caracterización y determinación del grado de peligro potencial está basado en la metodología desarrollada por Foster \& Hirata (1991), adaptada por el Proyecto SUWaR-MARENA-KTH, con la inclusión de un quinto parámetro. El método en su aplicación, utiliza el análisis de cinco características de la actividad contaminante.

1) Clase del contaminante involucrado en los procesos de contaminación; determinado por la degradación y retardación del contaminante con respecto al flujo del agua subterránea.

2) Intensidad del contaminante está definida por la relación de la concentración relativa y la proporción de la recarga afectada. La concentración relativa depende de la concentración del contaminante con respecto a las normas ABC Canadiense para la calidad del agua subterránea; dichas normas fueron utilizadas por el proyecto, ya que el método sugiere las guías de la OMS para la potabilización del agua.

3) El modo de disposición del contaminante en el sistema (suelo, subsuelo, agua) está relacionando a la carga hidráulica asociada al contaminante bajo la superficie a la 
que el efluente es descargado o donde la lixiviación de residuos sólidos ocurre. La carga hidráulica es la altura en mm/año de agua asociada con el contaminante que se infiltra hacia el subsuelo en un área de disposición. Duración de la carga que se evalúa por medio de la probabilidad de que el contaminante sea descargado al sistema, es decir, que se incorpore al suelo, para luego ser transportado e integrado al agua subterránea y el tiempo durante el cual se aplica la carga. La probabilidad es un criterio que se estima en base a la persistencia del contaminante y a la continuidad o constancia de aplicación del mismo.

5) Remediación del contaminante; este depende del volumen del contaminante y la posibilidad de remediación del mismo, de acuerdo a sus propiedades físico-químicas, como son densidad, solubilidad en el agua, degradación (con respecto a la bioremediación), volatilidad, cantidad, concentración y propiedades electroquímicas (SUWaR/NICARAGUA, 2000b).

El valor relativo de la carga se estima de la ponderación de las características descritas aplicadas a cada fuente, estableciéndose una escala relativa de peligro potencial de contaminación para cada tipo de actividad. Esta escala permite calificar la fuente como de bajo, moderado y alto peligro potencial de contaminación para las aguas subterráneas. El análisis para la determinación de la carga se basa en:

1) Identificación de fuentes contaminantes.

2) Priorización de fuentes contaminantes para su caracterización.

3) Caracterización de contaminantes.

4) Clasificación del peligro potencial.

\section{Identificación de fuentes contaminantes}

$\mathrm{El}$ inventario de fuentes contaminantes se realizó por medio de visitas a los sitios previamente identificados a través de la fotointerpretación, recorrido rápido de campo, uso de imágenes de satélite y de mapas topográficos; para esto se emplearon fichas o formatos, previamente diseñados, para cada fuente inventariada.
En el área de estudio se identificaron una gran variedad de actividades antropogénicas, como posibles fuentes potenciales de contaminación para las aguas subterráneas. Esta gran heterogeneidad de fuentes contaminantes inventariadas, se dividieron en cuatro grandes grupos de actividades agrícolas, industriales, municipales y otros.

Del inventario de fuentes contaminantes realizado en el área de estudio, se obtuvo un total de 590 fuentes según el tipo de actividad desarrollada. Revisada y analizada la información obtenida se seleccionaron 207 industrias entre grandes y medianas en las subcuencas occidental y central y 60 en la subcuenca oriental con el fin de verificar su ubicación y operación, de las cuales se clasificaron según la Clasificación Internacional Industrial Uniforme (CIIU) un total de 156 industrias (SUWaR/ NICARAGUA,2000b). Se verificó en el sitio las 207 industrias, donde se encontró que únicamente estaban operando 190.

Por las características generales del uso de la tierra en el área de estudio, la actividad que alcanza los mayores porcentajes es la actividad agrícola, sobre todo en las subcuencas oriental y occidental. El total de actividades agrícolas y actividades relacionadas con la agricultura identificadas fueron 148, entre las cuales se identificaron 102 áreas representativas de cultivos agrícolas entre las que se encuentran: granos básicos, piña, cítricos, hortalizas y café, en donde el $50 \%$ de estas representan probables fuentes de contaminación, por haber sido áreas utilizadas históricamente para el cultivo del algodón con un amplio uso de agroquímicos (SUWaR/NICARAGUA, 2000b). También se identificaron 16 actividades dedicadas totalmente a la ganadería, 23 actividades dedicadas a la agricultura y ganadería y 7 aeródromos.

Las actividades municipales identificadas corresponden a 589 áreas de desarrollo urbano de las cuales 20 son poblados, 18 comunidades, 550 urbanizaciones ubicadas en Managua y la ciudad de Masaya. Predominan las áreas de desarrollo urbano sin alcantarillado y con agua potable con un total de 368 fuentes solamente en la ciudad de Managua. Debido a la complejidad de la ciudad de Managua, se tomó la ciudad como una sola fuente de contaminación ya que el 55\% del área 
total es fuente generadora de contaminación por carecer de un sistema de alcantarillado y los tratamientos de aguas residuales existentes en su mayoría no cumplen con las especificaciones de diseño o están ubicados en lugares sensibles a la contaminación. De acuerdo a esta consideración, el total de áreas de desarrollo identificadas es de 40, siendo el $95 \%$ áreas urbana sin alcantarillado sanitario y con agua potable (SUWaR/NICARAGUA, 2000b).

Producto de la identificación e inventario de fuentes contaminantes a través de entrevistas en las principales compañías petroleras, visitas de campo en las fuentes de manera aleatoria para la verificación de la información e inspección de las mismas en el área de estudio se obtuvo un total de 75 estaciones de servicio, 16 hospitales y un aeropuerto internacional. Las actividades clasificadas como otras representan, un total de 92 fuentes, siendo las estaciones de servicio con abastecimiento de combustible las más representativas.

\section{Priorización de fuentes contaminantes}

Como resultado de la priorización de 590 fuentes contaminantes identificadas, se obtuvo que el $38 \%$ son de alta prioridad, $25 \%$ de mediana prioridad, $20 \%$ de baja prioridad y el $18 \%$ de ninguna prioridad. Esta priorización estableció la jerarquía o el orden de importancia con que debía realizarse el análisis de caracterización de las fuentes identificadas, según el tipo de actividad, áreas afectadas y el peligro potencial de los desechos generados.

\section{Caracterización de fuentes contaminantes}

La caracterización de las fuentes contaminantes se obtuvieron con base en el análisis de cada una de las cinco características conceptualizadas denominadas: clase, intensidad, modo de disposición, duración y remediación del contaminante. Este análisis se realizó para cada una de las fuentes contaminantes de forma particular e interrelacionando las características según el grado de influencia de cada una de ellas, para obtener un valor cualitativo que permita ubicarlas en alguna de las categorías: bajo, moderado o alto peligro potencial de contaminación.
El peligro potencial de contaminación representa el grado de peligro en un tiempo inmediato o futuro de las sustancias o compuestos que utilizan las fuentes en el desarrollo de la actividad y el tipo de efluente (líquido ó sólido) que genera cada una de ellas.

Actividad agrícola: por las características propias del área, se identificaron 125 áreas con terrenos agrícolas grandes y otros con pequeñas extensiones, en donde una gran cantidad tienen antecedentes de cultivo de algodón, ubicadas principalmente en las zonas comprendidas entre Veracruz, Cofradía, Sabana Grande, Altos de Masaya y ciudad Sandino, Los Brasiles y Mateare. Por las características mismas de las actividades agrícolas caracterizadas dentro del área de estudio, resultaron 36 actividades de alto peligro, 84 de moderado peligro y 28 actividades de bajo peligro potencial de contaminación.

En la caracterización de las actividades que resultaron de alto peligro potencial, contribuyeron principalmente las grandes áreas de cultivo agrícola donde se llevó a cabo una actividad de forma mecanizada, con gran cantidad y concentración de agroquímicos utilizados históricamente desde el auge del cultivo del algodón y que en la actualidad son grandes extensiones de tierra dedicados al cultivo de los granos básicos. Además, se incluyeron los aeródromos en donde se manipulaban y derramaban grandes cantidades de agroquímicos concentrados durante el cultivo del algodón tomándose en cuenta la clase, intensidad relativa, modo de disposición, tiempo de aplicación y remediación del contaminante (SUWaR/NICARAGUA, 2000b).

Actividades industriales: Las actividades industriales, después de las agrícolas, son las de mayor incidencia en la subcuenca oriental. Es muy común encontrar en esta zona granjas avícolas, industrias alimenticias, agroindustria (beneficios de café, trillos de arroz) en la parte alta de la subcuenca, donde se encuentran las mayores áreas de cultivo de café en la zona. En la parte media y baja de la cuenca, acercándose a la ciudad de Managua y Masaya, se presentan industrias más complejas y de alto peligro por el 
manejo de los desechos y el manipuleo de químicos. Las industrias de mayor peligro en el área son las que producen sustancias químicas o en su proceso utilizan compuestos químicos peligrosos, tóxicos y volátiles u otros compuestos venenosos.

Al contrario de la subcuenca oriental, en las subcuencas central y occidental las industrias que prevalecen pertenecen a diversas actividades, predominando la industria alimenticia y la química; las industrias como tal no son problemáticas, sin embargo, la materia prima y el mantenimiento de las maquinarias o para combustión utilizan hidrocarburos o derivados en grandes cantidades y son almacenados en tanques subterráneos. Estos se consideran de una probabilidad bastante alta para generar un gran peligro para las aguas subterráneas ya que generalmente están dispuestos en el subsuelo y en algunos casos el nivel freático está muy somero; al igual ocurre en las tuberías de los mismos.

En éste grupo de actividad industrial se contabilizaron 47 fuentes contaminantes de alto grado de peligro, definido por las características de mayor influencia: la clase de contaminante, el tiempo de aplicación de la carga, la posibilidad de remediación, eficiencia del proceso y las características físico-químicas de los posibles contaminantes (SUWaR/NICARAGUA, 2000b).

En las industrias de moderado peligro se contabilizaron un total de 48, entre las cuales se encontraron agroindustrias con utilización de desinfectantes y agroquímicos, producción agropecuaria, confección de prendas de vestir y jabonerías. Resultaron 94 industrias de bajo peligro siendo estas alimenticias, productos de plásticos, materiales de construcción, prendas de vestir, productos metálicos (fabricación de maquinarias menores a partir de hierro colado), agroindustria y producción agropecuaria las cuales no generan efluentes ni utilizan contaminantes peligrosos para las aguas subterráneas (SUWaR/MARENA, 2000b).

Actividades municipales: Las áreas de desarrollo urbano rural son fuentes que tienden a un alto peligro de contaminación para el sistema. De un total de 41 áreas de desarrollo urbano identificadas se encontraron 20 poblados con agua potable y sin ninguna cobertura de alcantarillado sa- nitario donde las aguas servidas son depositadas directamente en el subsuelo y en algunos casos en lugares donde el nivel freático es somero, lo que facilita una infiltración directa a las aguas subterráneas. Los grados de peligrosidad generados por estas situaciones varían de moderado a alto en dependencia del modo de disposición, clase del contaminante, concentración del contaminante y densidad poblacional.

En el área existe un total de 15 basureros de los cuales 5 son pequeños sin ninguna atención y el origen de los desechos son domésticos y dispersos en el área, por lo general están ubicados en áreas poblacionales, los cuales son considerados como basureros clandestinos, considerándose de bajo peligro potencial (Fig. 5). Los 10 restantes fueron considerados basureros municipales por sus grandes extensiones y la gran cantidad de disposición de desechos que en su mayoría son domésticos, los cuales se obtuvieron 8 de

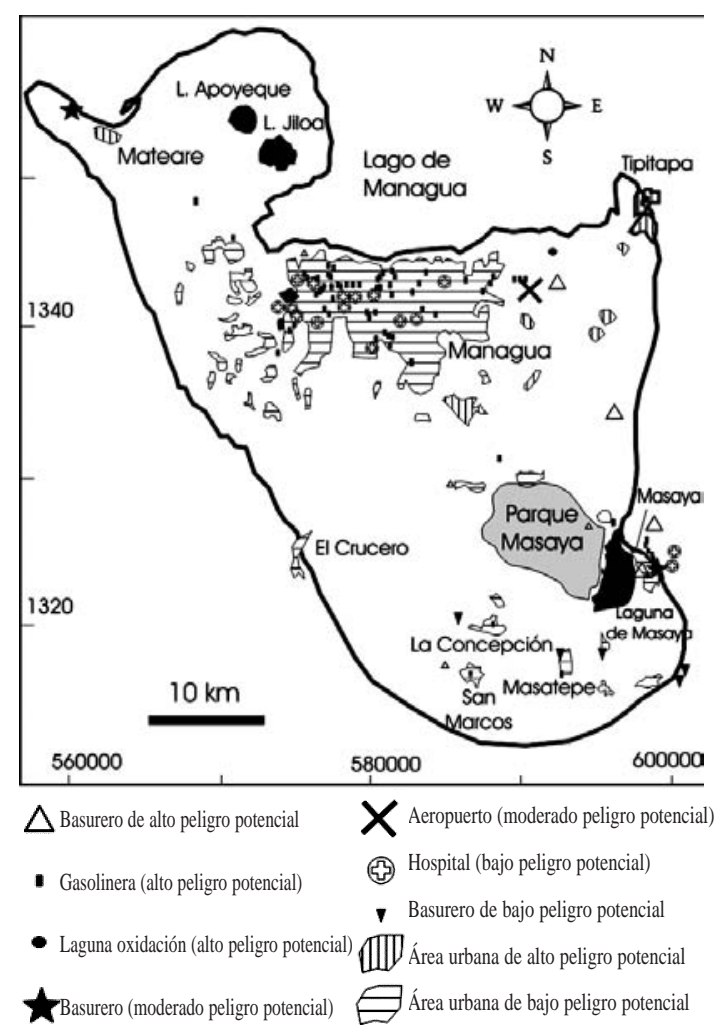

Fig. 5: Mapa de peligro potencial. 
alto peligro potencial y 2 de moderado peligro; sin embargo, en algunos de ellos existen desechos industriales debido a la cercanías de las industrias (SUWaR/NICARAGUA, 2000b).

Las fuentes de alto peligro se refieren a áreas urbanas con alta concentración y extensión poblacional, en donde no hay un manejo y disposición adecuada de los efluentes líquidos y sólidos, ya que utilizan sumideros, fosas sépticas, letrinas y basureros municipales para la disposición final de sus desechos. Además, hay basureros en donde depositan grandes cantidades de desechos sólidos y líquidos peligrosos de origen municipal e industrial, con condiciones constructivas inadecuadas para la disposición de desechos sólidos peligrosos.

\section{Mapa de peligro potencial de contaminación}

En condiciones similares al mapa de vulnerabilidad se elaboró un mapa temático para cada tipo de actividad, con el peligro potencial que cada fuente representa para el agua subterránea clasificadas en: alto peligro, moderado peligro o bajo peligro (Fig. 5).

\section{Riesgo potencial de contaminación del agua subterránea}

La información generada por la vulnerabilidad hidrogeológica y la generada por la carga contaminante es correlacionada para definir el riesgo potencial de contaminación de las fuentes inventariadas y caracterizadas. Esta correlación se hace a través de la matriz de riesgo (Cuadro 5).

El riesgo de contaminación se interpreta como la probabilidad de que las aguas subterráneas se contaminen, dependiendo de la interacción que ocurra entre la vulnerabilidad y la carga contaminante. Su determinación puede ser utilizada para estimar cuales fuentes identificadas ameritan una atención prioritaria por su alto riesgo potencial de contaminación a diferencia de aquellas que presentan un bajo riesgo. Esta prioridad permite enfocar los esfuerzos, los recursos y las acciones sobre aquellas fuentes que ameritan una mayor atención.

Las áreas de vulnerabilidad (alto, moderado y bajo), son áreas cuyas características y composición varía a largo plazo, por lo que se consideran áreas relativamente firmes para tomarlas como las áreas de protección. Por lo tanto, las áreas de protección del plan son las áreas de vulnerabilidad en el orden de alta, moderada y baja; y el orden de prioridad para las actividades, lo establece la condición de riesgo.

Esta zonificación del terreno en zonas muy vulnerables, zonas moderadamente vulnerables y zonas poco vulnerables a la contaminación de sus aguas, permite una discrecionalidad en la aplicación de regulaciones de protección; siendo más exigentes en las zonas muy vulnerables y menos exigentes en las zonas poco vulnerables, sin descuidar el peligro potencial de la actividad a través del concepto de riesgo. Este enfoque ayuda a evitar el establecimiento de regulaciones de prohibición en una amplia gama de actividades y zonas, que en determinado momento puede constituirse un fuerte obstáculo al desarrollo económico de una zona de protección. De esta manera se enfocan las acciones de prohibición o las más restrictivas solamente en aquellas actividades que impliquen un serio peligro de contaminación y en aquellas zonas en donde la vulnerabilidad a esa contaminación es muy alta.

Cuadro 5

Vulnerabilidad hidrogeológica

\begin{tabular}{llll}
\hline & Vulnerabilidad alta & Vulnerabilidad moderada & Vulnerabilidad baja \\
\hline Carga alta & Riesgo alto & Riesgo alto & Riesgo moderado \\
Carga moderada & Riesgo alto & Riesgo moderado & Riesgo bajo \\
Carga Baja & Riesgo moderado & Riesgo bajo & Riesgo bajo \\
\hline
\end{tabular}




\section{CONCLUSIONES}

1) El desarrollo de métodos ensayados en el área piloto del acuífero de Managua y los resultados obtenidos por el proyecto, son perfectamente reproducibles a mayores escalas en otros acuíferos del país.

2) El conjunto de herramientas obtenidas, constituyen un valioso aporte para la toma de decisiones relacionadas con los planes de protección de las aguas subterráneas.

3) El marco técnico generado por el proyecto, compuesto de la definición de las áreas de vulnerabilidad hidrogeológica del acuífero, la estimación del peligro potencial de contaminación de las actividades antropogénicas que se desarrollan en el lugar; el procedimiento para estimar el valor relativo de la protección del agua subterránea, son resultados que tienen su aplicación propia y que, además, pueden ser combinados entre sí para derivar otros métodos adicionales.

4) Los resultados obtenidos permiten preparar un plan de protección del acuífero que contenga directrices para la prevención y control de la contaminación de las aguas subterráneas ajustadas a la caracterización de los problemas encontrados.

5) La actualización de los métodos y los resultados obtenidos deben ser revisados cada tres o cinco años.

6) Las técnicas desarrolladas no deben volverse fines en sí mismo, sino que deben de ser utilizadas como herramientas en la toma de decisiones importantes.

7) El proyecto ha proveído capacitación y entrenamiento y ha generado un equipo de trabajo que está preparado para ejecutar los resultados y extender y replicar el trabajo en otros acuíferos.

\section{RECOMENDACIONES}

1) Un proyecto de esta naturaleza, destinado a desarrollar e implementar una estrategia de protección para las aguas subterráneas, debe ser ejecutado en el marco de una organización con mandato para institucionalizar sus resultados.

2) La aplicación de resultados debe insertarse en la perspectiva de desarrollar un plan nacional de protección de aguas subterráneas.

3) El desarrollo de métodos, las directrices de protección y los mecanismos de aplicación, deben hacerse con participación interinstitucional, principalmente, entre la autoridad ambiental, la instancia reguladora del uso de las aguas y las instancias encargadas del abastecimiento.

4) Introducir la planificación y la ejecución del plan de protección del acuífero, al enfoque de manejo integrado de cuencas hidrográficas.

5) Desarrollar un plan de monitoreo que permita un seguimiento a la evolución de la calidad de las aguas subterráneas del acuífero, como consecuencia de la aplicación del plan de protección.

6) Completar la estrategia de protección con estudios hidrogeológicos dirigidos a establecer posibles riesgos relacionados con la sobreexplotación del acuífero y con el valor del agua como un bien económico.

\section{REFERENCIAS}

ABT Associates Inc., 1995: Estudio de factibilidad del programa de manejo de la cuenca del lago de Managua. - 98 págs. Abt. Asssociates Inc. Managua.

ALLER, et al., 1987: DRASTIC. A standardized method for evaluating ground water pollution using hidrogeologic setting. - 428 págs. Oklahoma, U.S.A.

FOSTER, S. \& HIRATA, R. 1991: Determinación del riesgo de contaminación de las aguas subterráneas. - 81 págs. [ 2 ed.] Ed. CEPIS, Lima.

INEC, 2001: Instituto Nacional de Estadísticas y Censos. Compendio Estadístico 1990-1999. - 414 págs. Managua.

INAA-JICA, 1993: Proyecto de abastecimiento de agua en Managua. - 385 págs. Informe final, Managua. 
SUWaR/MARENA, 2000a: Mapa de vulnerabilidad hidrogeológica del acuífero de Managua. - 1/50000, Managua.

SUWaR/MARENA, 2000b: Mapa de peligro potencial de contaminación de la actividad municipal y otras actividades del acuífero de Managua. - 1: 50.000, Managua.

SUWaR/MARENA, 2000: Mapa del Acuífero de Managua. 1: 260.000, Managua.
SUWaR-NICARAGUA, 2000a: Vulnerabilidad hidrogeológica del acuífero de Managua. - 72 págs. Ministerio del Ambiente y Recursos Naturales (MARENA), Universidad Politécnica de Estocolmo (KTH).

SUWaR-NICARAGUA, 2000b: Estimación del peligro potencial de contaminación del acuífero de Managua. 115 págs. Ministerio del Ambiente y Recursos Naturales (MARENA). Universidad Politécnica de Estocolmo (KTH). 